\title{
Trivium
}

Revue franco-allemande de sciences humaines et sociales - Deutsch-französische Zeitschrift für Geistesund Sozialwissenschaften

$10 \mid 2012$

Lisibilité

\section{Mantique et herméneutique}

\section{Wolfram Hogrebe}

Traducteur : Pierre Rusch

\section{Q OpenEdition}

\section{Journals}

Édition électronique

URL : http://journals.openedition.org/trivium/4159

DOI : $10.4000 /$ trivium.4159

ISSN : 1963-1820

Éditeur

Les éditions de la Maison des sciences de l'Homme

Référence électronique

Wolfram Hogrebe, "Mantique et herméneutique », Trivium [En ligne], 10 | 2012, mis en ligne le 30 mars 2012, consulté le 08 septembre 2020. URL : http://journals.openedition.org/trivium/4159 ; DOI :

https://doi.org/10.4000/trivium.4159

Ce document a été généré automatiquement le 8 septembre 2020

\section{(c) (i) () $\Theta$}

Les contenus des la revue Trivium sont mis à disposition selon les termes de la Licence Creative Commons Attribution - Pas d'Utilisation Commerciale - Pas de Modification 4.0 International. 


\title{
Mantique et herméneutique
}

\author{
Wolfram Hogrebe \\ Traduction : Pierre Rusch
}

\section{NOTE DE L'ÉDITEUR}

Nous remercions Wolfram Hogrebe et la maison d'édition Königshausen \& Neumann pour nous avoir accordé l'autorisation de traduire cet article pour le présent numéro.

1 Il faut commencer avec deux dieux, Apollon et Hermès. Apollon est le dieu de la musique. C'est pourquoi il tient une lyre sous le bras. Mais il est aussi - cela a souvent été négligé, notamment par Nietzsche - le dieu de «la violence différée » (G. Colli). Car il tient dans l'autre main l'arc avec lequel il décoche les flèches qui répandent la peste, par exemple dans l'armée grecque devant Troie. Et il est enfin le dieu de la prophétie, le dieu de l'oracle de Delphes, le dieu de l'interprétation des signes naturels, qui révèlent le passé, le présent et le futur - il est donc aussi le dieu de la mantique.

2 Hermès, lui, est le dieu des voleurs, des troupeaux et des pasteurs, le dieu de la fécondité, le dieu des magiciens, des messagers et des interprètes. À ce titre, il est le dieu tutélaire de l'herméneutique.

3 Le fait que ces deux domaines de l'interprétation que sont l'interprétariat et la prophétie possèdent chacun son propre dieu montre que dans l'Antiquité la mantique et l'herméneutique étaient perçues comme deux domaines distincts. Il n'y a donc pas de place, du moins dans la conception antique, pour le projet d'une herméneutique universelle, tel que Hans-Georg Gadamer par exemple le revendiqua avec force au siècle dernier. Ou plutôt: il n'y aurait de place pour un tel projet que si l'herméneutique intégrait la mantique à la suite d'un « rachat hostile ».

Or c'est précisément ce qui s'est passé. Le processus de fusion s'achève au plus tard chez Georg Friedrich Meier (1718-1777). Au $\$ 256$ de son Essai d'un art universel de l'interprétation (Halle, 1757), on lit cette brève remarque : «L'art pratique universel de l'interprétation considère, outre le discours, d'autres genres de signes [...] Il peut s'agir de signes naturels ou arbitraires. Cet art enseigne donc à interpréter soit des signes 
annonciateurs, soit d'autres signes. Dans le premier cas, il s'appelle "art mantique d'interprétation" (hermeneutica mantica) ${ }^{1}$. "

Un deuxième témoignage de l'intégration de la mantique dans l'herméneutique nous est fourni par Friedrich Schleiermacher. Notre effort pour comprendre un texte consiste d'abord, selon cet auteur, à comparer un passage obscur avec d'autres. C'est ce qu'il appelle la "méthode comparative ». Or dans cette activité de comparaison, nous sommes censés à un moment donné voir soudain comment le passage en question doit être compris, comment ce passage particulier, d'abord impénétrable, peut être interprété à la lumière d'une idée générale et devenir ainsi herméneutiquement communicable. Cette opération d'interprétation éclairante "ne se produit», dit Schleiermacher, "que par divination $»^{2}$. Dans son discours à l'Académie du 12 août 1829, il emprunte explicitement à Platon ce concept de «divination». Le premier exposé sommaire de l'Herméneutique de 1819 recourait même au terme grec de " prophétique », en lieu et place du latin « divinatoire».

6 À son insu, Gadamer prolonge cette tradition d'une herméneutique qui a absorbé la mantique. On en trouve une belle expression à cet endroit de Vérité et méthode où il thématise spécifiquement la dimension universelle de l'herméneutique ${ }^{4}$. Gadamer explicite ici la constitution fondamentale de tout ce sur quoi la compréhension peut s'exercer par cette formule restée célèbre: "L'être qui peut être compris est langage ${ }^{5}$.» Le tournant «ontologique » revendiqué par Gadamer n'apparait donc possible, à plus proche examen, que sur la base d'une herméneutique élargie à la mantique, dans la tradition de Meier-Schleiermacher. C'est seulement ainsi qu'il parvient à étendre le concept de langage, de sorte que ce sont les choses elles-mêmes qui s'adressent désormais à nous : "C'est ainsi que nous parlons non pas seulement d'un langage de l'art, mais aussi d'un langage de la nature, d'un langage qui est celui des choses ${ }^{6}$.» Le langage devient pour Gadamer, comme pour Novalis, une "grande écriture chiffrée qu'on entrevoit partout: sur les ailes, la coquille des œufs, dans les nuages, dans la neige, dans les cristaux et dans la conformation des roches, sur les eaux qui se prennent en glace, au-dedans et au-dehors des montagnes, des plantes, des animaux, des hommes, dans les lumières du ciel, sur les disques de verre et les gâteaux de résine qu'on a touchés et frottés, dans les limailles autour de l'aimant et dans les conjectures singulières du hasard. On pressent que là est la clef de cette écriture merveilleuse, sa grammaire même; mais ce sentiment ne veut prendre aucune forme précise et arrêtée ${ }^{7}$ "

7 Le projet d'herméneutique universelle de Gadamer, avec son tournant ontologique, peut donc aussi se résumer dans la formule suivante : ici, Hermès ne s'est pas contenté de voler les vaches de son frère Apollon, il l'a supprimé purement et simplement.

8 Je ne dis d'ailleurs pas cela dans l'intention de discréditer ce projet, mais seulement pour en faire comprendre la spécificité. Gadamer ne défend assurément pas - Hasso Jäger a entièrement raison sur ce point - une herméneutique dans la tradition de l' Hermeneutica generalis de Johann Conrad Dannhauer (1603-1666), lequel avait entrepris d'enseigner à discerner, sans distinction de discipline, « le sens véritable du faux dans tous les discours et les écrits des auteurs » [verum sensum a falso discernere in omnibus auctorum scriptis et orationibus] $]^{8}$. Cette tradition fut plutôt invoquée dans les années 1990 par un certain nombre de jeunes auteurs soucieux justement de se démarquer de l'herméneutique gadamérienne, dont ils ne comprenaient pas la singularité que nous venons d'esquisser?. 
Ce sera la tâche d'une future histoire de la philosophie que de déterminer pourquoi cette époque en particulier a vu fleurir autant d'analyses consacrées à l'acte de compréhension et d'interprétation. Sans doute comprendre n'était-il pas devenu plus aisé en ce monde après 1989, à un moment où la perspective optimiste d'une fusion des horizons, telle qu'elle s'était dessinée dans les années 1960, se trouvait soudain confrontée au phénomène historique de leur dislocation - lequel semble constituer le véritable secret herméneutique de la mondialisation. Mais qui s'en rend compte? On se contente d'en subir les effets.

10 Comme nous l'avons dit, Gadamer se donna la possibilité d'associer à l'herméneutique une revendication d'universalité en y intégrant clandestinement la mantique. Ses critiques n'en savent rien, pas plus qu'il ne le sait lui-même. Les premiers dénoncent son projet, parce qu'il n'a pas grand-chose à voir avec la compréhension correcte de l' intentio auctoris, avec une analyse des conditions de réussite des interprétations correctes, et veut davantage: une analyse de la compréhension comme forme élémentaire de notre mode d'existence historique. Or c'est précisément ce qui est suspect aux yeux de ses premiers critiques, comme Emilio Betti, comme des plus récents.

11 C'est pourquoi j'essaierai de sauver au moins l'intention de Gadamer. Je le ferai en soulignant ce qui constitue un aspect de la compréhension qui ne se trouve pas assez mis en évidence chez certains représentants d'une herméneutique d'origine analytique.

12 Ceux-ci s'efforcent avant tout de donner une définition aussi serrée que possible [« das Brutto-Distinktionsprodukt zu erhöhen »] de cette difficile matière première, pour être sûr de bien savoir de quoi l'on parle. Axel Bühler dénombre ainsi dix-sept sous-catégories dans l'interprétation déclarative des textes ${ }^{10}$. Ce chiffre impressionnant atteste combien notre pratique de la compréhension textuelle est différenciée. Le point décisif pour notre projet n'est cependant pas la discussion critique de tels tableaux et leur comparaison avec d'autres, par exemple avec les dix degrés de compréhension des énoncés linguistiques chez Oliver Scholz ${ }^{11}$, mais la distinction fondamentale opérée entre l'interprétation déclarative et l'interprétation non déclarative. En s'appuyant sur Paul Thom ${ }^{12}$ et Jerold Levinson ${ }^{13}$, Axel Bühler oppose les formes déclaratives et les formes performatives de l'interprétation ${ }^{14}$. Il y a interprétation performative quand je joue un rôle sur scène ou quand j'exécute une œuvre musicale. Mais ce sont justement là les formes d'interprétation qui n'intéressent pas Axel Bühler. C'est pourquoi elles n'entrent pas dans le champ de ses réflexions ultérieures. Or ce choix n'est pas sans conséquence, dans la mesure où c'est précisément et seulement dans cette direction qu'il est possible d'accéder à une herméneutique qui, au-delà de la technique habituelle, envisage réellement la compréhension comme un mode élémentaire de la condition humaine. D'autres distinctions sont bien sûr nécessaires. L'interprétation performative n'a pas lieu seulement quand un comédien tient un rôle au théâtre, mais à chaque fois que quelqu'un endosse un rôle, par exemple dans l'exercice d'une charge ou d'une fonction. Ainsi, le maire de Berlin interprète le rôle de maire de Berlin par la manière dont il remplit sa fonction. Nous interprétons tous, et nous aussi philosophes, certaines possibilités professionnelles de l'existence dans le répertoire de notre société, par la manière dont nous remplissons notre rôle spécifique, en l'occurrence par la manière dont nous apparaissons comme philosophes dans un amphithéâtre, dans un cours ou un livre. Il est vrai qu'à la différence de l'interprétation performative d'une œuvre musicale ou d'une pièce de théâtre, l'interprétation se fait ici sans partition ni scénario. 
Nous ne disposons que d'une description de rôle à travers des critères de compétence et un statut légal, définissant ses tâches spécifiques et son champ d'application. Un autre genre d'interprétation performative est constitué par les formes dans lesquelles nous communiquons. Ici non plus, il n'y a pas de scénario, mais des exigences situationnelles, ainsi que des conditions grammaticales et pragmatiques de réussite. Les exigences situationnelles sont par exemple celles-ci : au cours d'une noce, l'invité interprète son rôle d'invité par la manière dont il communique. Cette interprétation diffère de celle que constituerait son mode de communication à l'occasion d'un enterrement, ou de celle d'un scientifique dans un laboratoire. Toutes les formes d'interprétation performative sont plus ou moins contingentes: certes, tous les rôles du répertoire social ne sont pas accessibles à mon interprétation performative, mais plusieurs généralement le sont. Le juriste aurait aussi bien pu devenir poète - on en connaît plus d'un exemple. En dehors de ces rôles, il existe cependant des formes d'interprétation performative très élémentaires, pour ne pas dire rigides, qui ne sont pas contingentes ou du moins pas de la même manière. Ces formes non contingentes d'interprétation performative sont celles qui sont immédiatement liées à notre existence "sur place " (on the spot), à notre perception du lieu où nous nous trouvons à ce moment précis, où nous agissons et jouons notre vie. Elles comportent une part de compréhension scénique $^{15}$. Nous atteignons seulement ici des formes d'interprétation ou de compréhension qui englobent l'ensemble des registres perceptifs de notre nature physique, depuis le réflexe jusqu'à l'humeur du moment, au sentiment ou au pressentiment. De telles formes d'interprétation performatives ne sont pas contingentes, au sens où nous ne pouvons fondamentalement nous en départir, et que nous ne pouvons pas non plus nous y faire remplacer, comme si nous interrompions la représentation d'une pièce de théâtre ou laissions notre rôle à un autre. Nous pouvons de même nous démettre d'une fonction, ou nous faire remplacer dans son exercice. Tout cela n'est pas possible pour notre compréhension situationnelle, si ce n'est par des démissions radicales et désespérées comme le suicide. La performance de notre situation dans le monde se confond donc avec la manière dont nous existons. C'est parce que ces manières d'exister sont toujours déjà des formes d'interprétation ou de compréhension ${ }^{16}$ qu'un projet comme celui de Heidegger dans Être et temps (c'est-à-dire le projet d'une herméneutique existentiale) est possible. Il n'y a là rien de mystérieux, même si cet usage du mot " herméneutique » est encore inhabituel, puisque les formes d'existence qui se déploient sur un plan subsémantique sont par nature d'ordre mantique. Heidegger, à juste titre, conçoit d'emblée l'exister de l'homme comme un Être imprégné de sens. Et il n'a pas moins raison de penser qu'il faut d'abord rechercher le sens de l'Être où une forme spéciale d'Être apparaît sous la forme d'un Être-sens [Sinn-Sein], c'est-à-dire chez l'homme, avec sa perception de l'existence axée sur le Moi. La première forme non construite, c'est-à-dire non inférentielle, de la sémanticité [Sinnartigkeit] de l'existence est notre constitution émotionnelle. Toute situation nous est sans aucun doute présente d'abord à travers les affects qu'elle suscite en nous. Heidegger intègre de manière méthodique cet état de fait comme une caractéristique de notre manière d'exister. À cet égard, on peut aussi parler d'un constat ontologique qui débouche sur le projet d'une existence ontosémantique. Nous rendons compte ainsi du fait que nous «existons " certes sur le plan physique en un point du temps et de l'espace, que nous "végétons » sur le plan physiologique en tant que nous sommes constitués d'un amas de cellules, mais que nous "vivons » toujours aussi sur le plan sémantique. Ce qui pour l'instant ne signifie rien de plus que le fait que 
nous sommes incapables de sortir du champ de nos tonalités émotionnelles. La première élucidation sémantique de notre compréhension scénique relève toujours des états d'humeur [Stimmungen] et des sentiments. Heidegger le dit sans ambages: "Sur un plan ontologique fondamental, nous devons en effet abandonner à la "simple disposition" [der bloßen Stimmung] le dévoilement premier du monde ${ }^{17}$." Il souligne ensuite que cette indication concernant la première élucidation sémantique de notre compréhension scénique "dans la vision instable, vacillante et capricieuse du "monde" $\aleph^{18}$ ne doit naturellement pas être confondue avec une "tentative pour livrer ontiquement la science au "sentiment" $~^{19}$. Heidegger pressent par où il pourrait donner prise à la critique: introduire les «sentiments" dans la théorie de la connaissance, c'est s'exposer au reproche d'« irrationalisme ».

13 Mais à ce propos les objecteurs présumés peuvent aujourd'hui encore s'inspirer de Leibniz, qui a su mieux que quiconque intégrer émotions et cognitions dans un continuum sémantique couvrant tout le champ de la signification implicite à la signification explicite. Ce panorama nous apprend que notre carrière épistémique commence dans tous les cas plus tôt que ne le suggère Robert Brandom dans sa théorie d'une raison inférentielle. Il est vrai que même cet auteur accorde que nous sommes des êtres de sentiment autant que des êtres de compréhension. Mais les sentiments ne sont pour lui que les déclencheurs de notre reconnaissance d'énoncés doxastiques matériellement consistants $^{20}$. Le sens subsémantique ou subpropositionnel des sentiments qui déterminent notre état d'humeur se trouve ainsi soustrait à l'analyse, ou assujetti sur un mode purement fonctionnel aux exigences de justification de l'agir langagier. Ce qu'il nous faut, c'est donc une théorie des formes informelles de savoir dans la tradition de Leibniz. Pour rendre justice à ces fragiles oscillations initiales de la compréhension du sens, nous pouvons de nouveau recourir au vocabulaire de la mantique, initialement adapté aux nuances d'une pratique interprétative en situation de risque. Aujourd'hui encore, nous n'interprétons pas de la même manière quand nous lisons un livre dans notre fauteuil, et quand nous cherchons à nous orienter dans l'obscurité, au milieu d'un environnement inconnu. Les bruits prennent dans ce dernier cas une tout autre valence, et influent puissamment sur notre comportement. Deux instances, plus précisément, prennent sens dans ces situations périlleuses. Ce sont, premièrement, les indices fournis par le vague arrière-plan visuel et sonore, desquels nous retirons un certain nombre d'informations; deuxièmement, le mélange de sentiments paniques qui agissent sur notre comportement.

Aussi distingue-t-on depuis l'Antiquité deux formes d'interprétation mantique. La première est appelée technique ou inductive, et consiste à interpréter correctement les signes naturels significatifs pour notre comportement dans une situation de risque. La deuxième forme est la mantique naturelle : elle interprète les émotions qui surgissent en nous et qui, lorsqu'elles se déchargent d'une manière totalement incontrôlée dans le rêve, l'ivresse ou l'extase, appellent à leur tour l'interprétation. La mantique technique ou inductive, vouée à l'interprétation de ces signes naturels qu'on appellera plus tard omina, était initialement basée sur une connaissance de la nature alimentée depuis des millénaires par les expériences faites dans un environnement favorable ou hostile. Cette sorte de mantique fait usage d'une pratique inductive excessivement élargie. C'est pourquoi Euripide considérait que le meilleur devin était celui qui tirait les meilleures conclusions. En tant que devin, Calchas était en même temps l'amiral de la flotte 
grecque, qu'il conduisit sur les côtes troyennes dia mantosyne, par la vertu de son art divinatoire.

Ainsi, la mantique inductive comportait d'authentiques contenus de savoir, auxquels nous devons d'ailleurs une bonne partie de nos connaissances actuelles - par exemple dans le domaine pharmaceutique et médical. Certes, cette forme de mantique tomba en discrédit lorsque ses adeptes perdirent contact avec le contenu, et ne développèrent plus leurs interprétations que par la voie canonique, c'est-à-dire en se basant sur des compilations et autres "Clés des songes ». Cette évolution se produisit dès l'Antiquité, comme nous l'apprennent les charges de Platon contre la mantique, ainsi que la critique explicite de Cicéron dans la deuxième partie du De divinatione. Pourtant, Platon lui-même recourt au vocabulaire de la mantique pour décrire la première découverte de l'Idée des Idées, c'est-à-dire d'un savoir concernant l'Idée du Bien. Chacun s'oriente sur ce qui lui parait bon. Il est donc avisé d'appeler "bien", dans un premier temps, seulement ce que «toute âme poursuit et [ce en vue de quoi] elle fait tout, dont elle soupçonne l'existence ${ }^{21}$. Il arrive même à Socrate de se désigner lui-même comme un

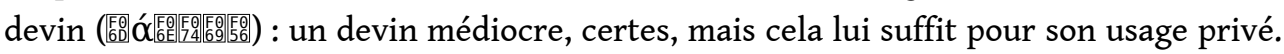
Il a coutume en effet de suivre la voix de son daimonion ${ }^{22}$, ce dernier vestige de la mantique naturelle qui se prolongera ensuite dans la période chrétienne sous la forme de la « voix de la conscience ». En ce sens atténué, chacun est à vrai dire un héritier des devins, et Socrate nous en indique la raison : "L'âme aussi, mon ami, est certainement douée d'une puissance divinatoire ${ }^{23}$.»

Malgré la critique sans ambiguïté adressée à la mantique professionnelle, qui avait déjà à son époque perdu tout sérieux scientifique et ne représentait plus qu'une pratique purement superstitieuse, Platon tenait encore la mantique pour un trait caractéristique de notre constitution psychique. Aujourd'hui encore, sans une part d'intuition divinatoire, nous ne saurions guère comment aborder les animaux, les hommes et le monde en général. D'un point de vue herméneutique, cette part mantique se répercute jusque dans notre communication quotidienne. Ce qu'un autre veut me dire, je commencerai toujours par le deviner avant de le savoir. C'est pourquoi la communication verbale exige toujours de la part de l'auditeur quelque chose comme une compréhension bienveillante. Il est significatif qu'un penseur comme Gottlob Frege ait montré un flair particulier pour ces formes de savoir informelles. À son grand dépit, il dut constater que même les notions de base qui sont nécessaires à la fondation logiciste des mathématiques échappent partiellement à la définition, parce qu'elles ont simplement un caractère élémentaire. Appartiennent malheureusement à cette catégorie des termes aussi importants que : vrai, bien, beau, mais aussi, non moins malheureusement, ceux de point et fonction. Si l'on veut introduire ces termes dans l'usage scientifique, en dépit de leur caractère non-définissable, il faut pouvoir compter, comme dit Frege, sur "une dose de bonne volonté, de compréhension bienveillante et d'intuition $»^{24}$. Du fait de cette indéfinissabilité, il existe donc aussi chez Frege des limites linguistiques de l'expression, qui ne sont pourtant pas des limites à la compréhension. Chez Frege aussi, notre compréhension dépasse notre compétence définitionnelle. Cela n'est d'ailleurs pas pour nous surprendre, dans la mesure où nous nous trouvons souvent embarrassés quand nous essayons de définir un mot difficile, bien que nous soyons bien sûrs de savoir ou de comprendre ce que signifie le mot en question. Dans la pratique quotidienne de l'intercompréhension, nous utilisons certains termes sans difficulté et nous parvenons à nous faire comprendre grâce à eux. Pourtant, si l'on nous interroge, nous avons du mal à en expliquer le sens. 
Celui qui entreprendrait par exemple d'expliquer des mots aussi banals que «tendre", " doux », "précautionneux ", " soigneux », ou encore " élégant », ou " balourd », ne s'en sortira pas sans exemples, sans anecdotes, sans images, et devra malgré tout comme l'a bien vu l'herméneute Frege - compter sur la compréhension bienveillante de son interlocuteur.

La même chose s'applique, selon Frege, à l'acquisition du langage par les nourrissons. "Avec eux, on doit pouvoir compter sur une entente bienveillante, de même qu'avec les animaux qui peuvent parvenir à une compréhension mutuelle avec l'homme ${ }^{25}$. " Pour cet auteur, les enfants et les animaux disposent donc déjà de ce sur quoi l'on doit au moins pouvoir compter : la capacité à comprendre et à se faire comprendre sur un mode prélinguistique. Ainsi se dessine un type de compréhension d'origine mantique, qui présente aussi une signification universelle pour l'herméneutique des rapports communicationnels.

Par la compréhension bienveillante, prise dans cette acception très large, nous conquérons un monde dans lequel tout, et chaque chose en particulier, devient un interlocuteur potentiel. C'est précisément un tel monde que visait Gadamer avec son projet d'une herméneutique universelle. Frege nous livre accès à ce monde dans lequel, selon la formule de Sir Edward Burnett Tylor (1832-1917), «le soleil et les étoiles, les arbres et les rivières, les vents et les nuages deviennent des créatures personnelles et animées ${ }^{26}$. Tylor qualifiait ce monde d' " animiste», un terme emprunté à la Theoria medica vera (Halle, 1707) de Georg E. Stahl (1660-1734). C'est seulement dans ce monde animiste que s'applique à strictement parler la thèse principale de Gadamer : "L'être qui peut être compris est langage». Dans un tel monde, des adultes peuvent communiquer avec des chiens, des enfants avec des poupées, des poètes avec la nature en général. Les adultes eux-mêmes sont durablement liés à ce monde animiste, du moins tant qu'ils restent réceptifs aux sollicitations de la nature, ce qui atteste que nous n'entretenons pas avec le monde seulement une relation active ou au contraire passive, mais aussi une relation médiale. L'histoire de la théorie de l'animisme jusqu'à Piage ${ }^{27}$ nous montre que sans une telle compréhension bienveillante, nous n'aurions pas construit le «Toi » obscur, et je voudrais suggérer ici que sans une dose minimale d'animisme, notre relation d'objet, c'est-à-dire notre capacité de référencialité ou d'intentionnalité, s'effondrerait. Car cette relation était à l'origine une relation personnelle, qui s'est transformée en relation d'objet lorsque le «Toi » obscur s'est enfoncé dans le mutisme. Les substituts techniques constituent une tentative désespérée pour lui rendre la parole. La technique est fille de l'animisme. Comme Pygmalion, elle commence par se fixer sur l'image, puis, accablée de solitude dans un monde muet, cherche à revenir en arrière.

Quel que soit le nombre d'espèces de compréhension que l'on puisse distinguer, toute compréhension explicite, c'est-à-dire énonçable sous forme propositionnelle, doit naître de sources non-propositionnelles, qui nourrissent aussi la genèse de notre relation d'objet. Au moins trois niveaux sont à prendre en considération dans ce contexte :

\begin{tabular}{|r|l|l|l|}
\hline 1. & $\begin{array}{l}\text { Affirmations/énoncés } \\
\text { propositionnels }(\mathrm{v} / \mathrm{f})\end{array}$ & $\mathrm{A}=\mathrm{F}$ & sémantique \\
\hline
\end{tabular}




\begin{tabular}{|r|l|l|l|}
\hline 2. & Conjectures/interprétations & $\begin{array}{l}\text { A peut être interprété } \\
\text { comme F }\end{array}$ & herméneutique \\
\hline 3. & Suggestions & A suggère F & mantique \\
\hline
\end{tabular}

a déjà distingué les deux premiers niveaux en opposant l'" en tant que [als] apophantique » à l'« en tant que herméneutique $»^{28}$. Il n'a pas identifié conceptuellement le troisième niveau, bien qu'il ne l'ignore pas sur le fond. En dessous de ce troisième niveau, le sens fait officiellement défaut. Ce niveau d'interprétation profond témoigne de notre capacité naturelle de résonance sémantique, que nous pouvons aussi appeler «mantique ».

Aidons-nous d'une image : si je fais tourner mon doigt humecté sur le bord d'un verre à vin, le cristal commence à chanter dès que le mouvement atteint une certaine régularité. C'est ainsi qu'il faut comprendre notre capacité de résonance médiale aux significations suggérées. Il arrive aujourd'hui que l'on analyse ces perceptions sous la dénomination de "qualia », mais ce terme malheureux n'a été choisi que pour réifier les suggestions et les orienter autant que possible vers une interprétation physicaliste.

Même le neurologue Antonio R. Damasio concède, il est vrai, que « le savoir commence par un sentiment $\aleph^{29}$. Mais, dans la mesure où il est incapable de traduire sur le plan neurobiologique des termes tels que « savoir » et « sentiment », cette phrase reste, chez lui aussi, une thèse philosophique.

Je crois qu'il nous faut, dans le projet d'une théorie neuronale de la connaissance, être beaucoup plus prudent que ne le sont certains. Il faut en tout cas prendre au sérieux la phrase suivante: «L'esprit n'est essentiellement que cela même qu'il sait de luimême ». Cette phrase est de Hegel ${ }^{30}$, et elle est vraie. Bien entendu, l'esprit sait aussi de lui-même qu'il ne sait pas tout et qu'il y a certaines choses qu'il ne peut pas savoir. Savoir et non-savoir vont donc de pair. Mais quel est l'écho de notre savoir renvoyé depuis la dimension du non-savoir?

\section{BIBLIOGRAPHIE}

Abel G. (1993) : Interpretationswelten, Francfort-sur-le-Main : Suhrkamp.

Abel G. (1999) : Sprache, Zeichen, Interpretationen, Francfort-sur-le-Main : Suhrkamp.

Brandom, R. (2000) : Making it Explicit, Harvard University Press ; trad. fr. : Rendre explicite, trad. I. Thomas-Fogiel, Paris : Éd. du Cerf, 2011 ; trad. all. : Expressive Vernunft, trad. E. Gilmer et H. Vetter, Francfort-sur-le-Main : Suhrkamp, 2000.

Bühler, A. (éd.) (1994) : Unzeitgemäße Hermeneutik. Verstehen und Interpretation im Denken der Aufklärung, Francfort-sur-le-Main : Klostermann.

Bühler, A. (1999) : « Die Vielfalt des Interpretierens », Analyse \& Kritik, 21, p. 117-137. 
Damasio, A. R. (2000) : « Eine Neurobiologie des Bewusstseins », dans : Newen, A. / Vogeley, K. (éds.) : Selbst und Gehirn, Paderborn : Mentis, p. 315-331.

Frege, G. (1906) : « Über die Grundlagen in der Geometrie », Jahresbericht der Deutschen Mathematiker-Vereinigung, 15 (6), p. 293-430.

Frege, G. (1969 [1924/25]) : « Erkenntnisquellen der Mathematik und Naturwissenschaften », dans : id. : Nachgelassene Schriften, éd. par H. Hermes, F. Kambartel et F. Kaulbach, Hamburg : Meiner, p. 286-294 ; trad. fr. : « Les sources de connaissance en mathématiques et sciences mathématiques de la nature ", dans : Écrits posthumes, trad. J.-P. Belna, Nîmes : Éd. Jacqueline Chambon, 1994, p. 315-323.

Frege, G. (1969 [1914]) : « Logik in der Mathematik », dans : id. : Nachgelassene Schriften, éd. par H. Hermes, F. Kambartel et F. Kaulbach, Hamburg : Meiner, p. 219-270 ; trad. fr. : « La logique dans les mathématiques ", dans : Écrits posthumes, trad. C. Thiercelin et F. Nef, Nîmes : Éd. Jacqueline Chambon, 1994, p. 241-296.

Gadamer, H. G. (1996) : Vérité et méthode, trad. coll., Paris : Le Seuil.

Hegel, G.W.F. (1988) : Encyclopédie des sciences philosophiques, III. La philosophie de l'esprit, trad. B. Bourgeois, Paris : Vrin.

Heidegger, M. (1986) : Être et temps, trad. F. Vezin, Paris : Gallimard.

Hogrebe, W. (2001) : Frege als Hermeneut, Bonn.

Lenk, H. (1993) : Interpretationskonstrukte, Francfort-sur-le-Main : Suhrkamp.

Levinson, J. (1993) : « Performative versus Critical Interpretation in Music », dans Krausz, M. (éd.) : The Interpretation of Music, Oxford : Oxford University Press, p. 33-60.

Looft, R. / Bartz, W. H. (1969) : « Animism Revived », Psychological Bulletin, 71, p. 1-9.

Lorenzer, A. (1970) : Sprachzerstörung und Rekonstruktion, Francfort-sur-le-Main : Suhrkamp.

Meier, G. F. (1965 [1757]) : Versuch einer allgemeinenen Auslegungskunst, rééd. par L. Geldsetzer, Düsseldorf : Stern-Verlag.

Novalis (1975) : Les Disciples à Sais, dans CEuvres complètes I, trad. A. Guerne, Paris : Gallimard.

Platon (1964), Phèdre, trad. E. Chambry, Paris : Garnier.

Platon (1966) : La République, trad. R. Baccou, Paris : Garnier.

Schleiermacher, F. (1987) : Herméneutique, trad. C. Berner, Paris : Éditions du Cerf.

Scholz, O. (1999) : Verstehen und Rationalität, Francfort-sur-le-Main : Klostermann.

Strube, W. (1985) : « Analyse des Verstehensbegriffs », Zeitschrift für Allgemeine Wissenschaftstheorie, XVI, p. 315-333.

Thom, P. (2000) : Making Sense. A Theory of Interpretation, Lanham : Rowman and Littlefield.

Tylor, E. B. (1871) : Primitive culture, Londres ; trad. fr. : La Civilisation primitive, trad. P. Brunet, Paris : Reinwald, 1876-1878.

\section{NOTES}

1. Meier (1965).

2. Schleiermacher (1987), p. 150. 
3. Schleiermacher (1987), p. 124.

4. Gadamer (1996), p. 500.

5. Ibid.

6. Ibid.

7. Novalis (1975), p. 37.

8. Cité d'après Scholz (1999), p. 40.

9. Je renvoie au recueil coordonné par Axel Bühler (1994), qui rassemble des travaux de Luigi Cataldi Madonna, Paolo Lombardi, Hans-Peter Schütt, Lutz Danneberg, Oliver Scholz, Gotfried Hornig et Mario Longo, sur l'herméneutique des Lumières. On peut aussi mentionner l'ouvrage d'oliver Scholz, cité dans la note précédente. Cet ouvrage a le mérite de confronter l'herméneutique aux théories de l'interprétation issues de la philosophie analytique. Si je m'en tiens ici à ces travaux qui, dans les années 1990, portaient explicitement sur l'herméneutique, cela ne suffit cependant pas à restituer tout l'arrière-plan que formaient à cette époque, significativement, les études philosophiques consacrées à la théorie de l'interprétation. Parmi celles-ci, il faut naturellement ranger les travaux de Günter Abel (1993; 1999), de Hans Lenk (1993) et déjà de Werner Strube (1985). Mentionnons enfin Paul Thom (2000), et surtout Robert Brandom (2000).

10. Cf. Bühler (1999).

11. Cf. Scholz (1999), p. 249 sqq.

12. Thom (2000), p. 54.

13. Levinson (1993), p. 33-60.

14. Cf. Bühler (1999).

15. La formule est utilisée ici dans un sens plus large que chez Alfred Lorenzer, qui parle de « compréhension scénique » pour caractériser la compréhension psychanalytique (cf. Lorenzer [1970], p. 104 sqq).

16. Cf. Abel (1993) ; (1999).

17. Heidegger (1986), p. 182 (trad. mod. ; soulignement WH).

18. Ibid. (trad. mod.).

19. Ibid. (trad. mod.).

20. Brandom (2000), trad. all., p. 400 sq.

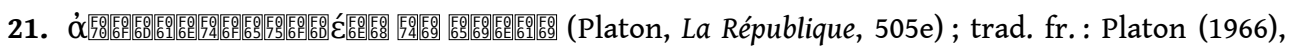
p. 265.

22. Cf. Platon, Apologie de Socrate, $31 \mathrm{c}$.

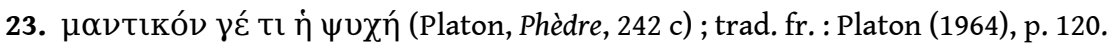

24. Frege (1906), p. 288. Cf. aussi Frege (1969[1914]), p. 224 ; trad. fr. Frege (1994), p. 246 (trad. mod.). Sur cette problématique chez Frege, cf. aussi Hogrebe (2001).

25. Frege (1969[1924/25]), p. 290 ; trad. fr. Frege (1994), p. 319.

26. Tylor (1871).

27. Contre la critique de Piaget, cf. Looft / Bartz (1969).

28. Cf. Heidegger, Être et temps, § 33.

29. Damasio (2000), p. 327.

30. Hegel (1988), p. 398 (§ 385, Additif). 
INDEX

Schlüsselwörter : Semantik, Gadamer, Einbeziehung der Mantik in die Hermeneutik, Frege, entgegenkommendes Verstehen

Mots-clés : sémantique, Gadamer, intégration de la mantique dans l'herméneutique, Frege, compréhension bienveillante

\section{AUTEURS}

\section{WOLFRAM HOGREBE}

Wolfram Hogrebe est professeur de philosophie à l'université de Bonn. Pour plus d'informations, lire la notice suivante. 